\title{
http://dx.doi.org/10.35381/racji.v5i8.575
}

\section{Ejercicio de la silla vacía como derecho de participación}

\section{Exercise of the empty chair as a right of participation}

\author{
Marjorie Anabelle Ortega-Romero \\ marjorie.ortega@ucacue.edu.ec \\ Universidad Católica de Cuenca, Cuenca \\ Ecuador \\ https://orcid.org/0000-0002-5680-752X \\ Cecilia Ivonne Narváez-Zurita \\ inarvaez@ucacue.edu.ec \\ Universidad Católica de Cuenca, Cuenca \\ Ecuador \\ https://orcid.org/0000-0002-7437-9880 \\ Enrique Eugenio Pozo-Cabrera \\ epozo@ucacue.edu.ec \\ Universidad Católica de Cuenca, Cuenca \\ Ecuador \\ https://orcid.org/0000-0003-4980-6403 \\ Juan Carlos Erazo-Álvarez \\ jcerazo@ucacue.edu.ec \\ Universidad Católica de Cuenca, Cuenca \\ Ecuador \\ https://orcid.org/0000-0001-6480-2270
}

Recibido: 13 de noviembre de 2019

Aprobado: 14 de diciembre de 2019

\section{RESUMEN}

Investigación relativa al Derecho Constitucional reflejado en la figura de participación ciudadana por medio del mecanismo de la silla vacía en Ecuador. Se ha evidenciado falta de interés ciudadano por hacer uso del mecanismo e incluso se refleja su escaza documentación en los casos que sí pudo ser ejecutado, creando controversia en torno al uso de la silla vacía, quien debería ocuparla y las normativas que deberían regular su proceso en los GADs. Por lo mismo, se ha propuesto que el objetivo de estudio sea analizar mediante la revisión de la literatura especializada el ejercicio de la silla vacía como derecho de participación y mecanismo para lograr la satisfacción de las necesidades colectivas en el Ecuador. 
Para ello se ha empleado una metodología de tipo bibliográfica-documental, la misma que permitió la revisión y análisis de teorías, leyes, procesos e investigaciones en relación al tema central para lograr un análisis reflexivo.

Descriptores: Derecho Constitucional; Participación ciudadana; Silla vacía; Gobiernos autónomos descentralizadas; Democracia.

\begin{abstract}
Research related to Constitutional Law reflected in the figure of citizen participation through the empty chair mechanism in Ecuador. There has been evidence of lack of citizen interest in making use of the mechanism and even its scarce documentation is reflected in the cases that could be executed, creating controversy regarding the use of the empty chair, who should occupy it and the regulations that should regulate its process in the GADs. For this reason, it has been proposed that the objective of the study be to analyze, through the review of the specialized literature, the exercise of the empty chair as a right of participation and mechanism to achieve the satisfaction of collective needs in Ecuador. For this, a bibliographic-documentary methodology has been used, which allowed the revision and analysis of theories, laws, processes and research in relation to the central theme to achieve a reflexive analysis.
\end{abstract}

Descriptors: Constitutional Right; Citizen participation; Empty chair; Decentralized autonomous governments; Democracy.

\title{
INTRODUCCIÓN
}

En Ecuador el Derecho Constitucional se orienta a estudiar los aspectos más importantes que rigen en las instancias políticas estatales y su concordancia con los derechos y garantías fundamentales como lo explica Salgado (1996) quien además indica a éste como parte del Derecho Público en cuanto se refiere a consideraciones muy particulares del Estado vigilando el adecuado funcionamiento de esta organización para el desarrollo del pueblo por medio de un grupo de leyes.

Al respecto, Calderón (2015) afirma que dichas leyes regulan muchos de los procesos estatales, entre los cuales figura el derecho a la participación que permite la actuación democrática del poder político a través de la expresión libre y voluntaria de la ciudadanía que le otorga legitimidad a su accionar. Por lo tanto, la participación ciudadana se constituye en un derecho constitucional amparado en la 
carta magna y les permite a las personas, expresar su voluntad en relación a aspectos de orden público.

Incluso, la participación ciudadana en su intento de explicar una dinámica social, es considerada como un derecho que puede modificar las relaciones de poder y permitir la generación de una conciencia crítica y propositiva por parte de los ciudadanos. En términos de Álvares (2014) se trata de una acción continua que involucra a los ciudadanos ya sea de manera individual o en un colectivo para la toma de decisiones, fiscalización, monitoreo y cumplimiento de gestiones públicas y privadas de orden político, económico, social y ambiental de forma que intervenga en su progreso y desarrollo como individuo y como parte de un contexto social Guanipa Ramírez, Albites Sanabria, Aldana Zavala \& Colina Ysea, 2019).

Por lo antes expuesto, uno de los recursos participativos más importantes en el contexto ecuatoriano, la silla vacía, mecanismo diseñado para que los ciudadanos puedan intervenir en las sesiones de sus gobiernos locales aportando con ideas, opiniones o planteamientos estructurados acerca de las temáticas que atañen a su contexto, potenciando mejoras para la comunidad. Hecho que en el Ecuador se encuentra amparado en la ley por medio de la Constitución de la República (2008) mediante la cual se fortalece la participación ciudadana en busca de la toma de mejores decisiones por parte de las autoridades sectoriales, locales y provinciales como resultado de una relación colaborativa entre la sociedad y el Estado. Por ello se han incorporado competencias nuevas para los Gobiernos Autónomos Descentralizados (GADs), integrando la participación ciudadana y la electoral.

La competencia participativa de la ciudadanía establece garantías para que los ciudadanos puedan ejercer su derecho a intervenir en la gestión pública, sobre todo para fiscalizar y combatir actos corruptos, otorgándoles la potestad de protagonizar la toma de decisiones estatales y de la sociedad, ello como parte de un proceso continuo para la construcción del poder ciudadano. Es entonces cuando se instaura la silla vacía como oportunidad para ser parte de los debates y la toma de decisiones, involucrando la participación, representación y deliberación de todos los actores de la sociedad. 
Sin embargo, en contraposición a lo expuesto, en la actualidad la figura jurídica de la silla vacía no se ha ejecutado de manera efectiva y los ciudadanos no han reconocido ni asumido la relevancia que tiene este derecho, por lo que se plantea como objetivo de investigación el analizar mediante la revisión de la literatura especializada el ejercicio de la silla vacía como derecho de participación y mecanismo para lograr la satisfacción de las necesidades colectivas en el Ecuador, pues en las investigaciones al respecto se puede ver como no es usado homogéneamente en los distintos cantones, en tal razón se destaca la siguiente interrogante ¿Cómo garantizar la participación ciudadana en los diferentes niveles de Gobierno como mecanismo para lograr la satisfacción de las necesidades colectivas?.

\section{Participación ciudadana en Ecuador}

En el Ecuador existen diferentes formas de participación ciudadana, las mismas que se evidencian a través de las constituciones a lo largo de la historia, pues en cada una de ellas se ha procurado la definición de cuáles son los espacios para que los ciudadanos puedan ser partícipes en temas de interés para los gobernantes además de que se cumpla con el ejercicio de la democracia con transparencia.

Zúñiga (2015) refiere que a final de los años sesenta e inicios de los setenta, el sector agrario demandó la necesidad de ser reconocidos como movimiento social, quienes procuraban defender sus intereses debido a la amenaza latente sobre sus propiedades y es que hasta dicho momento el único derecho de participación ciudadana registrado era el del voto. Posterior a ello, a inicios de los noventa, fue el pueblo indígena quien marco los procesos de participación ciudadana a nivel nacional, cuyo protagonismo político se sustentó en el concepto de comunal, representado por movimientos del área andina y amazonia.

Luego en 1998, se incorporaron en la constitución otros mecanismos de participación como lo registra Zúñiga (2015) entre los cuales está la consulta popular, derogatoria de mandato, iniciativa pública para la manifestación de proyectos de ley y derechos de orden político, siendo así como se inicia de forma decisiva en el fomento para que la ciudadanía tome parte activa en las decisiones 
de gobierno. Para el año 2008 la constitución reflejaba que la participación ciudadana era reconocida como un derecho de los ciudadanos el cual debía ejercerse en todo ámbito, siendo además el principio bajo el cual las instancias públicas debían actuar, de forma que se garantizara la existencia de espacios y procesos de consulta por parte de las personas.

Y es a partir de la última reforma constitucional en el año 2008 que la participación ciudadana, de acuerdo con Pérez (2015) es comprendida como el derecho de las personas por intervenir protagónicamente en la toma de decisiones y gestión pública, además de tener un carácter de conciencia colectiva del pueblo, ante factores que impiden su desarrollo. Es así como se establecen mecanismos participativos tales como audiencias públicas, cabildos populares, silla vacía, veedurías, observatorios y consejos de consulta, además de la consulta previa. Estos mecanismos promueven el accionar ciudadano por medio de la reflexión crítica y de procesos que potencien estructuras organizativas en busca del bienestar común. Así, en el Art. 95 de la Constitución de la República, se indica:

Los ciudadanas y ciudadanos, en forma individual y colectiva, participarán de manera protagónica en la toma de decisiones, planificación y gestión de los asuntos públicos, y en el control popular de las instituciones del Estado y la sociedad, y de sus representantes, en un proceso permanente de construcción del poder ciudadano. La participación se orientará por los principios de igualdad, autonomía, deliberación pública, respeto a la diferencia, control popular, solidaridad e interculturalidad. La participación de la ciudadanía en todos los asuntos de interés público es un derecho, que se ejercerá a través de los mecanismos de la democracia representativa, directa y comunitaria (Constitución de la República del Ecuador, 2008).

Sin embargo, Rizzo (2010) indica que los diferentes grupos sociales conformados en países, ciudades, comunidades, etc., no son partícipes en los procesos de vinculación con sus gobiernos debido a la falta de conciencia de este derecho y por el uso de los mecanismos participativos como estrategia burocrática para la presentación de planes de desarrollo, es decir que el involucramiento ciudadano se ha transformado en un recurso netamente formal o con intereses políticos de ciertos sectores. Pese a lo expuesto, en el contexto ecuatoriano, la participación ciudadana 
ha sido una condición indispensable para alcanzar una democracia real y con ella lograr un verdadero beneficio para el pueblo.

Esto se puede evidenciar no solo en sus condiciones de progreso de algunos sectores que han sabido hacer uso efectivo de su derecho participativo, también existen un gran grupo de ciudadanos cuyos comportamientos rutinarios, frente a problemáticas comunes como la inseguridad, salud, entre otros, están marcados por el desinterés y la poca participación social para erradicarlos. $Y$ por otra parte, se encuentran aquellos casos en los que se ha hecho uso de la silla vacía bajo la influencia de colectivos, empresarios o actores políticos para fines particulares.

\section{La silla vacía}

La silla vacía es un mecanismo de participación ciudadana que forma parte de la gestión pública, se trata de un instrumento para que los ciudadanos ya sea en forma individual o como parte de un colectivo, puedan participar activamente en los diferentes niveles de gobierno que establece la ley. Este recurso forma parte de las sesiones públicas de los Gobiernos Autónomos Descentralizados, caracterizado por ser un espacio participativo en el que uno o varios representantes ciudadanos tomaran un rol activo frente a la toma de decisiones en cuanto a temas de interés, tal como lo indica la Ley Orgánica de Participación Ciudadana.

Por su parte, Bovero (2012) se refiere a la silla vacía como uno de los mecanismos diseñados para lograr la participación ciudadana que fue propuesto para que los individuos y grupos sociales formen parte activa en el debate y puedan ser partícipes en la toma de decisiones de los gobiernos locales dando valor a su voz y valorando su capacidad de votar. Es por ello, que Ramírez y Espinosa (2012) insisten en que la silla vacía debe ser analizada como figura en el proceso de participación ciudadana debido a su doble connotación, por un lado, adquiere una perspectiva deliberativa y por otro como representación social.

Sin embargo, el aspecto más importante que se debe tomar en cuenta según Scherz (2013) hace referencia al derecho del ocupante de la silla vacía para ejercer el voto, ya que esta atribución invalida la voluntad del pueblo, quien previamente ha elegido a sus mandantes en un proceso considerado universal, además se registra 
una transgresión en lo que se refiere a la equidad democrática por que este se fundamenta en excluir las divergencias de tipo arbitrario de la política.

Para solucionar esta divergencia Noguera (2013) considera que se debe estructurar el proceso de participación a través de la silla vacía por medio de una regulación en la que no se excluya a ningún sector y se permita al ciudadano que haga uso de ella, exponer su posición y ser partícipe del debate público sin ejercer votación alguna, con la finalidad de obtener un espacio para deliberar sin que exista intervención política anticipada y se pueda articular a los conformantes del sistema gubernamental bajo principios de igualdad y democracia.

Se entiende entonces que la silla vacía es un mecanismo al que las personas pueden acceder ya sea por decisión propia o como representantes de un colectivo por previa elección de sus miembros y no por la votación popular de la comunidad a la que pertenecen, por lo que quien la ocupe no se caracteriza por ser un actor de algún movimiento político y será quien propicie la participación democrática y el ejercicio de los derechos ciudadanos como figurantes en espacios públicos específicos.

\section{La silla vacía en Sudamérica}

La silla vacía como mecanismo de participación ciudadana también se encuentra en las Constituciones de Uruguay, Argentina, Perú, Chile, Venezuela y Bolivia, fue configurada como proceso de transformación democrática. No obstante, Francini y Castaño (2018) manifestaron que, en Venezuela y Bolivia, existen hallazgos importantes en términos de participación ciudadana, esto se debe a que la evolución política ha venido marcada por cambios de tipo institucional que cuestionaron el clasismo de sus democracias por lo que se adoptaron reformas constitucionales.

En relación a ello, Partlow (2019) explica estas actualizaciones como una revolución de papel por cuanto se refieren a modificaciones en la estructura jurídico-política. Cabe mencionar que tanto Venezuela y Bolivia en los últimos años han acarreado una marcada crisis de tipo económico e incluso colapso en el ámbito político (Peña y Lillo, 2019). Estos elementos que generaron trances en los países mencionados, 
incentivaron que sus Estados sean refundados por lo que se instauraron mayores controles por parte del Gobierno respecto a la participación ciudadana.

Peña y Lillo (2019) sugieren que el poder popular en los países Sudamericanos, actúa como un canal para mediar entre el cumplimiento de la democracia y la comunidad, por lo que la silla vacía se convierte en un instrumento participativo y protagónico para los grupos sociales externos al contexto político. Al respecto, Chaguaceda (2018) indica que la participación ciudadana en Venezuela y Bolivia pueda terminar como una contribución pragmática debido a que el Estado es quien promueve la organización ciudadana y la conduce conforme sus fines, por lo que los gobiernos seccionales en cada caso, son el eje central de los procesos de participación, de manera que pierde valor y protagonismo la iniciativa de las personas.

\section{La silla vacía en Ecuador}

La silla vacía fue incorporada en la Constitución del Ecuador, y en su artículo 1, se hace mención a la posibilidad que tienen los ciudadanos de ser parte de los debates y toma de decisiones y también se acota sobre la participación ciudadana por medio de representantes (Constitución de la República del Ecuador, 2008). Esto se refuerza en el artículo 77 de la Ley de Participación Ciudadana y Control Social donde se estable que las personas podrán participar en las reuniones locales como representantes de la ciudadanía ya sea de manera individual o colectiva dependiendo de la temática a tratar.

Por otra parte, en el Código Orgánico de Organización Territorial, Autonomía y Descentralización se establece en su artículo 13 que las reuniones de los GADs serán de tipo público y deberán tener en ellas el espacio para la silla vacía, pudiendo esta ser ocupada por un ciudadano para su participación conforme el tema del día e incluso gozando de la capacidad de votar, rigiéndose a las normativas legales fijadas previamente (Asamblea Nacional, 2010). Sin embargo, en el contexto nacional, de acuerdo con lo expuesto por Ramírez y Espinosa (2012) los distintos gobiernos locales no cuentan con una reglamentación que norme el ejercicio de la 
silla vacía, y regule la selección de las personas que representarán a la comunidad en los espacios dentro de las sesiones de consejo.

Esto pese a que en la Ley Orgánica de Participación Ciudadana (2010) en su artículo 77 explica que la elección de los participantes ciudadanos debe darse en reuniones de orden popular o público, considerando que el representante intervendrá en una sesión del GAD respecto a un tema en particular, por lo que cada instancia será la responsable de establecer los respectivos parámetros para validar a quienes soliciten el recurso de la silla vacía sin que esto sea una limitante en el ejercicio del derecho participativo sino más bien una normativa para su adecuada ejecución.

Al respecto, la Procuraduría General del Estado (2011) ha reconocido que las facultades del representante de la silla vacía serán las mismas que se le atribuyen a un funcionario de gobierno, pero realizando una clara y contundente excepción en la cual se detalla que el ciudadano no podrá participar en la toma de decisiones políticas si no, netamente en asuntos de interés general, reforzando el artículo 77 de la Ley Orgánica de Participación Ciudadana antes mencionado en lo que respecta a las intervenciones ciudadanas en temas específicos. Queda claro entonces que la participación de los ciudadanos en la silla vacía puede ser orientada a una intervención de tipo colaborativa, por ejemplo, para elaborar un plan local de política pública, temas relacionados con el presupuesto u otras gestiones públicas relacionadas a su comunidad, entre otros.

Ante lo expuesto, es preciso aclarar que, en cada intervención, el individuo solo podrá participar considerando la temática para la que fue citado. Pese a esto, el mayor problema se encuentra en la falta de interés ciudadano por ejercer este derecho participativo a lo que se le atribuyen distintos factores, entre los que se mencionan el desconocimiento de su función y el beneficio que podría otorgar a un colectivo y la comunidad en general, además de la poca confianza de las personas en la gestión y transparencia en los GADs.

Situación que se ha comprobado en diferentes investigaciones, ejemplo de ello es la de Zúñiga (2015) quien explicó que en la ciudad de Cuenca no existe involucramiento de las personas para el uso de la silla vacía, además se pudo 
constatar que los individuos desconocían respecto a este derecho y que las autoridades locales no lo socializaban. De igual manera Jiménez (2016) constató que los ciudadanos del Cantón Francisco de Orellana no participaban activamente en las sesiones de concejo e incluso se determinó su desconocimiento respecto a los derechos que tienen en relación a la participación ciudadana y específicamente en lo referente a la silla vacía.

Otro ejemplo es el de Congo (2017) en el Cantón Bolívar en cuya indagación se estableció que las autoridades locales vulneraban el derecho de participación ciudadana en cuanto no institucionalizaron los procesos para que se regulara la figura de la silla vacía. También se suman los aportes de Pacheco (2017) con la investigación en las ciudades de Latacunga y Salcedo, en la cual se concluyó que existió una total falta de conocimiento respecto a la silla vacía por parte de sus comunidades, la cual iba de la mano con la ausencia de adecuados procesos de comunicación y socialización por parte de las autoridades.

Esta breve revisión permite identificar que el derecho constitucional de la participación ciudadana a través de la silla vacía no ha sido ejecutado adecuadamente en los Gobiernos Autónomos Descentralizados de diferentes localidades en el Ecuador, evidenciando que la participación de la ciudadanía no sucede como se espera. Al respecto, Ramírez y Espinosa registraron que "apenas se aprobó el nuevo texto referido a la silla vacía, se multiplicaron las demandas ciudadanas por hacer uso del nuevo mecanismo" (2012, p. 120). Surgieron solicitudes de los ciudadanos por hacer uso de la figura de silla vacía a lo largo de todo el país que inicialmente no contaba con las condiciones necesarias para su ejercicio (tiempos, espacios, modos, requisitos, etc.).

Por lo que, no se pudo determinar cuáles fueron las intervenciones en el debate político que realmente contribuyeron a dotar de mayor racionalidad a la toma de decisiones de los gobiernos locales e incluso registrar si efectivamente el recurso de la silla vacía fue usado correctamente. La única consideración siempre fue la obligación, de las autoridades seccionales de publicar, con la debida anticipación, la convocatoria y la agenda de las sesiones locales para que la ciudadanía tuviese 
conocimiento de ella sin que esto represente una invitación a participar en las reuniones (Ramírez y Espinosa, 2012, p. 130).

Posterior a ello, la investigación de Castro (2014) infiere que hasta el año 2013 el derecho de la silla vacía presentaba una disparidad entre los diferentes GADs a nivel nacional, por ejemplo, en la ciudades de Cuenca este derecho fue ejercido en 43 ocasiones de los cuales solo 4 personas hicieron efectivo su capacidad para votar; en Portoviejo se registraron solo 4 casos; en Ambato existieron 240 ocasiones en las que se lo ejerció; en Manta se registraron solo 3 veces, en relación a la provincia de Cañar el único dato encontrado se refiere a la existencia de 2 veedurías ciudadanas hasta el año 2015.

Sin embargo, Carrera (2015) reconoce que ha existido participación de las personas en el uso de la silla vacía, no obstante, su involucramiento se realiza sin la presencia de un reglamento interno que contenga las directrices para su ejecución y en todos los casos no se registra el seguimiento e información de las resoluciones tomadas y aplicadas por las autoridades a los intervinientes, evidenciando nuevamente una falta de normativas y parámetros a seguir por parte de los GADs.

Por consiguiente, no se encuentran evidencias de si dichas personas gozaron del suficiente conocimiento respecto al ejercicio de su derecho constitucional, solamente se conoce de manera general de acuerdo con Herrera (2013) que la mayoría de ciudadanos que acudieron a la silla vacía fueron "representantes de instituciones constructoras, inmobiliarias, financieras, o bien, terratenientes urbanos, quienes tienen intereses particulares. Sobre todo, porque la ciudadanía no conoce el mecanismo ni la tramitología burocrática para ocupar la silla vacía” (p. 17).

Ante ello, Arce y Herz (2014) consideran que la participación de la ciudadanía puede lograrse partiendo del diagnóstico de las problemáticas de la comunidad, sus necesidades y la búsqueda de recursos para solventarlas, además de la formulación de propuestas de vinculación entre el colectivo y las instituciones gubernamentales pertinentes. En dicho contexto, resulta estratégico que la comunicación sea considerada como un elemento innegable en el dinamismo social, pues esta facilita la participación de las personas en los procesos de desarrollo local, por lo que, si esta vinculación sucede bajo un sistema organizado, los ciudadanos podrán ser 
parte en la toma de decisiones que les compete, para la mejora de sus condiciones de vida y por ende de progreso.

Por su parte, Curnill (1997) expresó que "la potencialidad de la participación ciudadana para romper con los monopolios de poder está vinculado en la medida en que ella se guíe por los principios de la igualdad, del pluralismo político, y de deliberación" p. 300. Esto da continuidad a lo expuesto por Dahl (1956) quien manifiesta la existencia de cinco criterios que deben ser satisfechos por el proceso de gobierno a fin de que las personas que conforman una comunidad tengan igualdad de derechos en la participación de la toma de decisiones políticas que la afecten.

- Participación efectiva: la cual implica que todas las personas tengan igualdad de oportunidades para exponer sus ideas y propuestas respecto a cómo se debe hacer la política.

- Igualdad de voto: es decir que cada individuo tiene la oportunidad de votar.

- Comprensión ilustrada: esta hace referencia a que las personas gozarán de las mismas oportunidades para capacitarse en distintos aspectos relativos a su participación política.

- Control de asuntos: que consiste en que los integrantes de una comunidad puedan decidir acerca de los aspectos que debe contener la agenda.

- Inclusión social: referida a que todas las personas, indistintamente de su edad, género, raza, situación económica u otros aspectos, deben ser considerados como ciudadanos con pleno goce de sus derechos.

Pacheco (2017) indica que la efectividad de la figura de la silla vacía se fundamenta en la existencia de tres aspectos:

- La planificación a través de la colaboración de los órganos centrales y locales.

- La representación de la sociedad civil a través de las organizaciones sociales.

- La adecuada reglamentación para el acceso y uso de la silla vacía.

En este punto, se puede entender que la problemática nacional gira en torno a una inadecuada regulación de los mecanismos de participación ciudadana en la figura de la silla vacía como Derecho Constitucional, entonces queda claro que la falta de 
definición de la figura de la silla vacía ha generado varios debates y quizá uno de los más álgidos es aquel que se refiere a quién debe ocuparla.

Por ello, los autores citados en las páginas que anteceden, expresan que la dinámica participativa a través de la silla vacía y la designación de los representantes ciudadanos debe estar sujeta a una normativa local en la que se establezcan aspectos convenientes para cada nivel gubernamental y se regularicen los términos de intervención de la ciudadanía. Lo cual obligaría a los Gobiernos Autónomos Descentralizados a expedir regulaciones ad hoc para normar la utilización ciudadana de la silla vacía, que fijaría las condiciones y límites de su activación.

Ahora bien, existen evidencias de que el proceso de participación ciudadana a través del mecanismo de la silla vacía, debe ser llevado a cabo por medio de la construcción de un engranaje amplio de instituciones ciudadanas de toma de decisión, sobre todo una Asamblea de Unidad Cantonal, un Consejo de Desarrollo y Gestión y los Comités Intersectoriales de Trabajo, ya que, de acuerdo con Ramírez y Espinoza (2012) esta red de órganos participativos son los que han permitido que realmente se integre a la ciudadanía en la deliberación y gestión de lo público, de manera que sería importante integrar a los Consejos Consultivos que están dispuestos en el Art. 100 de la Constitución del Ecuador.

\section{METODOLOGÍA}

La metodología empleada en el presente artículo de revisión integró métodos, técnicas e instrumentos que se aplicaron a lo largo de la investigación, entre otros, el método jurídico el mismo que se empleó para analizar el ejercicio de silla vacía como derecho de participación ciudadana; el método inductivo - deductivo permitió analizar la participación ciudadana y su posterior regulación en los Gobiernos Autónomos Descentralizados, llegando así a conclusiones generales de la temática estudiada; el método de análisis de contenido facultó el examen objetivo, coherente y sistemático de las variables de estudio mediante la revisión de actas, artículos científicos, libros y memorias a fin de establecer inferencias, valoraciones cualitativas, y los alcances dogmáticos, académicos y legales vigentes, concluyendo 
que existe inconsistencias en la normativa legal vigente en Ecuador que regula este proceso (Saquicela, Erazo y Narváez, 2019).

\section{APORTES}

El ejercicio de la silla vacía como derecho de participación y mecanismo para lograr la satisfacción de las necesidades colectivas en el Ecuador ha procurado incentivar el involucramiento de la ciudadanía en el contexto público. Por lo que su enfoque se ha centrado en la ejecución de una actividad democrática pluralista en la que se destaca la importancia y necesidad de contar con la opinión y perspectiva de distintos colectivos y de los ciudadanos como impulsores para una mejor práctica gubernamental y acciones democráticas eficaces que a más de ello gocen de transparencia.

Sin embargo, en el ejercicio de este mecanismo se ha precisado la necesidad de considerar que las distintas agrupaciones sociales se caracterizan por aspectos diferentes lo que obviamente expresa una variedad de situaciones que pueden presentarse en torno a la participación de los ciudadanos, por lo que es muy importante que cada GAD analice la complejidad y extensión de la intervención ciudadana a fin de establecer normativas y que el proceso resulte como una actividad organizada que finalmente pueda ser de valor para ambas partes. Así los representantes de la comunidad también podrán tomar acciones propias conforme lo demande su contexto.

De esta manera el funcionamiento de la silla vacía se enfocará en alcanzar un consenso social por medio de la inclusión de un actor no tradicional, pero con capacidad de contribuir efectivamente en la toma de decisiones y en la ejecución transparente de los procesos públicos. Todo ello considerando que no todas las problemáticas demandan la participación ciudadana y que no muchas personas gozan del conocimiento necesario para el abordaje y solución de algunas situaciones y que no toda la ciudadanía puede participar directamente.

Es por ello, que resulta cuestionable el voto que actualmente pueden ejercer los ciudadanos que ocupan la silla vacía, ya que este puede incentivar la participación y también desembocar en un comportamiento autoritario y monopolizador cuando se 
involucran o están vinculados movimientos dominantes. Situación que pone de manifiesto nuevamente la necesidad de analizar la legitimidad de los ciudadanos que representan a corporaciones o con intereses especiales y que pretenden incurrir en una decisión colectiva ya que esta acción pone en duda el principio democrático que rodea al mecanismo. Por consiguiente, se reitera la importancia de normar el proceso de participación ciudadana a través de la silla vacía conforme las regulaciones de los GADs en su accionar público y la necesidad de contar con la ciudadanía en determinados temas para llevar a cabo un proceso eficaz, tal como se muestra en la figura 1.

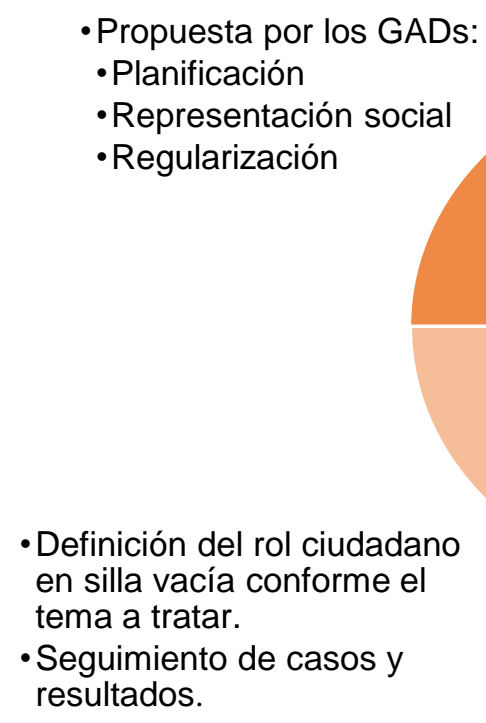

-Propuesta por los GADs:

Planificación

-Representación social

-Regularización

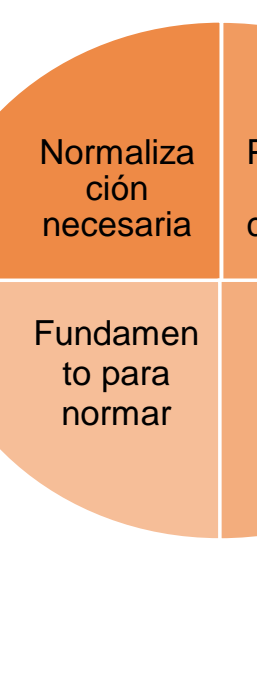

-Actuación democrática del poder político.

- Expresión libre y voluntaria de la ciudadnía.

Participaci

ón ciudadana

Silla vacía

Figura 1. Normalización del proceso de participación ciudadana - silla vacía-

\section{REFERENCIAS CONSULTADAS}

1. Álvarez, C. (Septiembre de 2014). Mecanismos de participación ciudadana para la planificación y evaluación de la gestión pública en el Ecuador. Margen(74). Recuperado el 28 de Junio de 2018, de https://www.margen.org/suscri/margen74/alvarez.pdf

2. Arce, R., \& Herz, W. (2004). De Excluidos a Protagonistas: El desafío de promover capacidades locales. (RICDL, Ed.) Lima: Red Perú. Recuperado el 1 de Diciembre de 2017 
3. Asamblea Nacional. (2010). Código Orgánico de Organización Territorial, Autonomía y Descentralización. Quito: Presidencia de la República. Recuperado el 28 de Junio de 2018, de http://www.oas.org/juridico/pdfs/mesicic4_ecu_org.pdf

4. Carrera, A. (2015). Estrategia jurídico informática para potenciar la participación ciudadana en los procesos de gobernabilidad en los entornos municipales. Universidad Regional Autónoma de los Andes, Facultad de Jurisprudencia. Ambato: UNIANDES. Recuperado el 28 de Junio de 2018, de http://dspace.uniandes.edu.ec/bitstream/123456789/592/1/TUAABG0662015.pdf

5. Castillo, J. (2008). Descentralización del Estado y articulación del espacio nacional, la regionalización horizontal, Ecuador=equidad (Cuarta ed.). Loja. Recuperado el 28 de Junio de 2018

6. Castro, J. (2014). La silla vacía como mecanismo de participación ciudadana. Pontificia Universidad Católica del Ecuador, Facultad de Jurisprudencia. Quito: Pontificia Universidad Católica del Ecuador. Recuperado el 28 de Junio de 2018 , de http://repositorio.puce.edu.ec/bitstream/handle/22000/11296/La\%20silla\%20v ac\%C3\%ADa\%20como\%20mecanismo\%20de\%20participaci\%C3\%B3n\%20c iudadana.pdf?sequence $=1$

7. Congo, A. (2017). Anteproyecto de ordenanza sobre el derecho fundamental del uso de la silla vacía en el Gobierno Autónomo Descentralizado del cantón Bolívar. Universidad Regional Autónoma de los Andes, Facultad de Jurisprudencia. Ibarra: Universidad Regional Autónoma de los Andes. Recuperado el 28 de Junio de 2018, de /

8. (2008). Constitución de la República del Ecuador. Quito: Asamblena Nacional. Recuperado el 12 de Enero de 2018, de http://www.inocar.mil.ec/web/images/lotaip/2015/literal_a/base_legal/A._Cons titucion_republica_ecuador_2008constitucion.pdf

9. Dahl, R. (1956). A Preface to Democratic Theory. Chicago: The University of Chicago Press. Recuperado el 22 de Marzo de 2018, de http://webs.ucm.es/info/cpuno/asoc/profesores/lecturas/dahl.pdf

10. Gallegos, F., \& Espinoza, A. (2012). Ocupando la silla vacía. Representación y participación en el tránsito posconstitucional del Ecuador. CENDES. Quito: CENDES. Recuperado el 28 de Junio de 2018

11. Guanipa Ramírez, L., Albites Sanabria, J., Aldana Zavala, J., \& Colina Ysea, F. (2019). Educación para la ciudadanía y la democracia: El equilibrio del 
$\begin{array}{llll}\text { poder. IUSTITIA } & \text { SOCIALIS, } & 4(6), & \text { 71-89. }\end{array}$ doi:http://dx.doi.org/10.35381/racji.v4i6.290

12. Hernández, R., Fernández, C., \& Baptista, P. (2010). Metodología de la investigación (Quinta ed.). México D.F.: Mc Graw Hill. Recuperado el 28 de Junio de 2018, de https://www.esup.edu.pe/descargas/dep_investigacion/Metodologia\%20de\%2 0la\%20investigaci\%C3\%B3n\%205ta\%20Edici\%C3\%B3n.pdf

13. Herrera, M. (28 de Octubre de 2013). Funcionario de la Dirección Técnica de la Asociación de Municipalidades del Ecuador. (J. Castro, Entrevistador) Quito: Pontificia Universidad Católica del Ecuador. Recuperado el 28 de Junio de 2018, de http://repositorio.puce.edu.ec/bitstream/handle/22000/11296/La\%20silla\%20v ac\%C3\%ADa\%20como\%20mecanismo\%20de\%20participaci\%C3\%B3n\%20c iudadana.pdf?sequence $=1$

14. Jiménez, J. (2016). Incidencia de la figura jurídica de la silla vacía dentro del derecho de participación ciudadana en el Gobierno Autónomo Descentralizado Municipal de Francisco de Orellana en el año 2014. Universidad Central del Ecuador, Facultad de Jurisprudencia, Ciencias Políticas y Sociales. Quito: Universidad Central del Ecuador. Recuperado el 28 de Junio de 2018, de http://www.dspace.uce.edu.ec/bitstream/25000/6501/1/T-UCE-0013-Ab237.pdf

15. López, A. (2008). Análisis del Referéndum Constitucional 2008. Iconos Revista de Ciencias Sociales(33), 13-20. Recuperado el 28 de Junio de 2018, de http://www.flacso.org.ec/docs/i33lopez.pdf

16. Naranjo. (1990). Teoría constitucional e instuciones políticas (Tercera ed.). Bogotá: Editorial Temis. Recuperado el 28 de Junio de 2018, de http://webcache.googleusercontent.com/search?q=cache:CWVaJRp70RMJ:si dn.ramajudicial.gov.co/GetFile.ashx\%3FpFileName\%3DpDUvi2LMX9U37\%2 52B15VQsCKozxRcsjsNl1actoNw2Ko7uQ0\%252BxUUpGsDPnxfKhWqgPIP wiYdCER1byq01qgcZ2hNRjUY0ecGgROT0ixZsnZq0BSGasIT7\%252FsK1XH gXp90

17. Pacheco, J. (2017). La participación ciudadana usando la silla vacía para las decisiones y debates en las sesiones legislativas en el Gobierno Autónomo Descentralizado Municipal de los cantones Latacunga y Salcedo. Pontificia Universidad Católica del Ecuador, Escuela de Jurisprudencia. Ambato: Pontificia Universidad Católica del Ecuador. Recuperado el 28 de Junio de 2018, http://repositorio.pucesa.edu.ec/bitstream/123456789/1845/1/76348.pdf 
18. Ramírez, F. (2012). Neoliberalismo, estado y cambio político (Tema Central). Revista Corriente Alterna No.01, 13-18. Recuperado el 28 de Junio de 2018, de http://repositorio.flacsoandes.edu.ec/handle/10469/3730\#.WzhjJdJKjlU

19. Rizo, M. (2010). La vigencia de La incomunicación de Carlos Castilla del Pino, 40 años después. Lecturas y reflexiones sobre intersubjetividad y la (in)comunicación. Mediaciones Sociales, II(7), 3-20. Recuperado el 22 de Marzo de 2018, de file://C:/Users/hp/Desktop/22275-22294-1-PB.PDF

20. Saquicela Rodas, V. H., Erazo Álvarez, J. C., \& Narváez Zurita, C. I. (2019). Modelo de Gestión estratégica para las Mipymes que conforman el Pasaje Artesanal Azuayo. Koinonía, 220.

21.Zayas, M. (2010). El rombo de las investigaciones de las Ciencias Sociales. Holguín: Universidad de Holguín. Recuperado el 28 de Junio de 2018, de http://biblioteca.utec.edu.sv/siab/virtual/elibros_internet/55708.pdf

22.Zúñiga, R. (2015). La silla vacía como nueva forma de participación ciudadana en el Municipio de Cuenca. Universidad de Cuenca, Facultad de Jurisprudencia y Ciencias Políticas y Sociales. Cuenca: Universidad de Cuenca. Recuperado el 28 de Junio de 2018, de http://dspace.ucuenca.edu.ec/bitstream/123456789/21596/1/TESIS.pdf 